Research Paper

\title{
Targeting Sphingosine Kinase by ABC294640 against Diffuse Intrinsic Pontine Glioma (DIPG)
}

\author{
Lu Dai" ${ }^{1 \#}$, Jungang Chen" ${ }^{1 \#}$, Zhen Lin", Zhaoxiong Wang'2, Shengyu $\mathrm{Mu}^{3}$, and Zhiqiang Qin ${ }^{1 凶}$ \\ 1. Departments of Pathology, Winthrop P. Rockefeller Cancer Institute, University of Arkansas for Medical Sciences, 4301 W Markham St, Little Rock, AR 72205, USA. \\ 2. Department of Pathology, Tulane University Health Sciences Center, Tulane Cancer Center, 1700 Tulane Ave., New Orleans, LA 70112, USA. \\ 3. Pharmacology \& Toxicology, Winthrop P. Rockefeller Cancer Institute, University of Arkansas for Medical Sciences, 4301 W Markham St, Little Rock, AR 72205, USA. \\ \#These authors contributed equally to this work. \\ $\triangle$ Corresponding author: University of Arkansas for Medical Sciences, 4301 W Markham St, Little Rock, AR 72205, USA. Phone: (501) 526-8619. E-mail: \\ zqin@uams.edu (Dr. Zhiqiang Qin).
}

(C) The author(s). This is an open access article distributed under the terms of the Creative Commons Attribution License (https://creativecommons.org/licenses/by/4.0/). See http://ivyspring.com/terms for full terms and conditions.

Received: 2020.03.23; Accepted: 2020.05.11; Published: 2020.05.22

\begin{abstract}
As a highly aggressive pediatric brainstem tumor, diffuse intrinsic pontine glioma (DIPG) accounts for $10 \%$ to $20 \%$ of childhood brain tumors. The survival rate for DIPG remains very low, with a median survival time as less than one year even under radiotherapy, the current standard treatment. Moreover, over than 250 clinical trials have failed when trying to improve the survival compared to radiotherapy. The sphingolipid metabolism and related signaling pathways have been found closely related to cancer cell survival; however, the sphingolipid metabolism targeted therapies have never been investigated in DIPG. In the current study, the anti-DIPG activity of $A B C 294640$, the only first-in-class orally available Sphingosine kinase (SphK) inhibitor was explored. Treatment with $A B C 294640$ significantly repressed DIPG cell growth by inducing intracellular pro-apoptotic ceramides production and cell apoptosis. We also profiled ABC294640-induced changes in gene expression within DIPG cells and identified many new genes tightly controlled by sphingolipid metabolism, such as IFITMI and KALI. These genes are required for DIPG cell survival and display clinical relevance in DIPG patients' samples. Together, our findings in this study indicate that targeting sphingolipid metabolism may represent a promising strategy to improve DIPG treatment.
\end{abstract}

Key words: sphingolipid; ceramide, brain tumor, DIPG, pediatric cancer

\section{Introduction}

Diffuse intrinsic pontine glioma (DIPG) is a highly aggressive pediatric brainstem tumor that accounts for about $10 \%$ to $20 \%$ of childhood brain tumors [1-3]. These tumors are mostly seen in children between 5 and 10 years old, but have been reported to occur at any age of childhood. The survival rate for DIPG remains very low, with a median survival time as less than one year even under radiotherapy, the current standard treatment. Moreover, more than 250 clinical trials have failed when trying to improve the survival compared to radiotherapy [4-6]. For example, some studies have reported chemotherapy has failed to show benefits beyond radiotherapy for improving DIPG patients' survival [5]. Moreover, the sensitive location of these tumors and lack in surgical specimens (or even lack in the patient-derived cell lines) have hindered our understanding of the DIPG development, pathogenesis as well as discovery of effective targeted therapy. We and others recently have reported some new therapeutic targets and agents against DIPG, including natural products (e.g., Brefeldin A, Combretastatin A4); Emtansine conjugated to peptide nanofiber precursor (DM1NFP); synthesized novel platinum (ii) complexes; combined AXL and HDAC inhibition [7-12]. However, most of these new treatments are still at preclinical stage, which require clinical trials to assess their efficacy and tolerability. Unfortunately, a very recent Phase 2 study reported that addition of valproic acid (VPA) and bevacizumab to radiation failed to improve the survival of DIPG patients [13].

The ceramidase in sphingolipid metabolism is responsible for hydrolytic conversion of ceramide to sphingosine, which is then phosphorylated by SphK1 
or SphK2, two isoforms of sphingosine kinases to generate sphingosine-1-phosphate (S1P) [14]. In fact, the cellular balance of ceramide and S1P can determine the fate of tumor cells, with the accumulation of ceramides favoring cell apoptosis, while the accumulation of S1P favoring cell proliferation $[14,15]$. Among sphingolipid metabolic, ceramide has been considered as a central lipid mediator with tumor suppression function, because it can tightly regulate many cellular signaling related to apoptosis, cell cycle and autophagy $[14,16]$. Due to their pleiotropic roles, targeting bioactive sphingolipids has recently evolved as a promising therapeutic approach for improving cancer treatment [17]. As a key enzyme of sphingolipid metabolism, a highly selective small molecule inhibitor of SphK, 3-(4-chlorophenyl)-adamantane-1-carboxylic acid (pyridin-4-ylmethyl)amide (named as ABC294640), has been recently developed [18,19], and shows significant anti-tumor effects on a variety of cancers [20-24]. Moreover, Britten et al. have reported the promising results from a Phase I clinical trial about ABC294640 in patients with advanced solid tumors [25]. They reported that $A B C 294640$ at $500 \mathrm{mg}$ bid was well tolerated by these cancer patients and achieved biologically relevant concentrations in their plasma [25].

Our group reported that targeting sphingolipid metabolism with either ABC294640 or exogenous ceramides resulted in in vitro and in vivo anticancer activities for virus-associated malignancies [20,26-28], as well as non-small cell lung cancer (NSCLC) [29]. However, the functional role of sphingolipid metabolism and related cellular network in DIPG remains almost unknown. Even ABC294640 has displayed broad anti-tumor activities in a variety of cancers, we think that the underlying mechanisms especially sphingolipid related cellular contents are tumor type-dependent. It is also unclear whether the sphingolipid metabolism targeted therapies can be developed for improving DIPG treatment. In the current study, we investigated the response of DIPG cells to SphK inhibition by ABC294640, identified new cellular genes controlled by sphingolipid metabolism in DIPG cells and validated their functions in DIPG pathogenesis. Our results provide new insights into the mechanism and potential utility of targeting sphingolipid metabolism in a deadly form of pediatric cancer.

\section{Materials and Methods}

\section{Cell culture and reagents}

The DIPG cell lines SF8628 and SF7761 that harbor the histone H3.3 Lys 27-to-methionine (K27M) mutation were purchased from Millipore-Sigma and cultured as recommended by the manufacturer. The cortical neuronal cell-line, $\mathrm{HCN}-2$, was purchased from American Type Culture Collection (ATCC), and cultured as recommended by the manufacturer. All the experiments were carried out using cells harvested at low $(<20)$ passages. ABC294640 was purchased from SelleckChem.

\section{Cell proliferation and apoptosis assays}

Cell proliferation was determined by using the WST-1 assays (Roche) according to the manufacturer's instructions. Briefly, after the period of treatment of cells, $10 \mu \mathrm{L} /$ well of cell proliferation reagent, WST-1 (4-[3-(4-Iodophenyl)-2-(4-nitrophenyl)-2H-5-tetrazolio]-1,3-benzene disulfonate), was added into 96-well microplate and incubated for $3 \mathrm{~h}$ at $37^{\circ} \mathrm{C}$ in $5 \% \mathrm{CO}_{2}$. The absorbance of samples was measured by using a microplate reader at $490 \mathrm{~nm}$. Flow cytometry was used to the quantitative analysis of apoptosis with the FITC-Annexin V/propidium iodide (PI) Apoptosis Detection Kit I (BD Pharmingen).

\section{Soft agar assays}

The anchorage-independent growth ability was determined using the soft agar assays as described previously [30]. Briefly, a base layer containing $0.5 \%$ agarose medium and 5\% FCS was poured into the six-well plates. Then, 2,000 cells were mixed with 0.4\% agarose in Dulbecco's Modified Eagle Medium (DMEM) containing $5 \%(\mathrm{v} / \mathrm{v})$ FCS to form a single-cell suspension. The plates were then incubated for $4-5$ weeks at $37^{\circ} \mathrm{C}$. Colonies were stained with $0.005 \%(\mathrm{w} / \mathrm{v})$ crystal violet and photographed under a phase-contrast microscope.

\section{Immunoblotting}

Total cell lysates $(20 \mu \mathrm{g})$ were resolved by $10 \%$ $(\mathrm{w} / \mathrm{v})$ SDS-PAGE, transferred to nitrocellulose membranes, and immunoblotted with antibodies for cleaved Caspase3, cleaved PARP, Bax, XIAP, SphK1, SphK2 (Cell Signaling), IFITM1, KAL1 (Abcam) and $\beta$-Actin or Tubulin (Sigma) for the loading controls. Immunoreactive bands were identified using an enhanced chemiluminescence reaction (PerkinElmer), and visualized by the autoradiography.

\section{Sphingolipid analyses}

Quantification of sphingolipid species was performed using a Thermo Finnigan TSQ 7000 triple-stage quadruple mass spectrometer (Thermo Fisher Scientific). Quantification was based on calibration curves generated by spiking an artificial matrix with known amounts of target standards and an equal amount of the internal standard. The ratio of 
sphingolipid normalized to total phospholipid phosphate level was shown as final results using the Bligh and Dyer lipid extract method as described previously [31].

\section{RNA-Sequencing and analysis}

RNA-Sequencing for each sample was performed in biological triplicate using the Genome Analyzer IIx (Illumina) at LSUHSC Translational Genomics Core (TGC) Facility. The completed RNA-Sequencing data has been submitted to NCBI Sequence Read Archive and available (SRA, accession \# PRJNA565990). Raw sequencing reads were analyzed using the RSEM software (version 1.3.0; human GRCh38 genome sequence and annotation) for quantification of human gene expression as previously described.25 The EBSeq software was utilized to analyze statistically differentially expressed genes using a false discovery rate (FDR) < 0.05. Differentially expressed genes between the ABC294640- and vehicle-treated cells were used as input for the Ingenuity Pathway Analysis (IPA)'s downstream effects analyses including the canonical pathway analysis and disease function analysis. The Z-score was calculated by the Z-score algorithm of the IPA, which can predict the direction of a biological function change. RNA-Sequencing data of DIPG tumor samples and paired normal brain tissues were obtained from the National Institutes of Health (NIH) Genotypes and Phenotypes ( $\mathrm{dbGaP}$ ) database under the accession number (SRA\# SRP136329) after obtaining the NIH permission. Transcript quantification of human genes was conducted as previously described [32].

\section{RNA interference (RNAi) assays}

For RNAi assays, IFITM1, KAL1, SphK1 or SphK2 On-Target plus Smart pool siRNA (Dharmacon) or negative control siRNA were delivered at the concentration of 10 or $25 \mathrm{nM}$, using the DharmaFECT transfection reagent as recommended by the manufacturer.

\section{Statistical analysis}

The two-tailed Student's t-test was used to determine the significance for differences between the experimental and control groups.

\section{Results}

\section{ABC294640 displays effective anti-DIPG activity}

As mentioned above, currently there are limited patient-derived DIPG cell lines commercially available. Here we used two DIPG cell lines, SF8628 and SF7761, both of which are derived by surgical biopsies from H3.3K27M DIPG patients [33]. H3.3K27M, a somatic mutation of histone H3.3 resulting in a lysine 27 to methionine substitution occurs in $60 \%$ of DIPG [34]. In H3.3K27M DIPG patient samples, the levels of H3K27 dimethylation (H3K27me2) and trimethylation (H3K27me3) are reduced globally. These epigenetic changes are thought to be important factors driving DIPG oncogenesis [34, 35]. In a time-course "killing" assay, we found that $\mathrm{ABC} 294640$ treatment effectively reduced SF8628 cell growth in a dose-dependent manner when compared to the vehicle-treated controls (Figure 1A). We also observed similarly inhibitory effects of ABC294640 on SF7761 cell line (Figure S1). In contrast, ABC294640 showed much less inhibitory effects on the growth of normal brain/neuronal cell-line, $\mathrm{HCN}-2$, with only $80 \mu \mathrm{M}$ concentration showing limited inhibition (Figure S2). Even though both DIPG cell lines carrying H3.3K27M, they display distinct morphology in cultures, especially SF7761 having much less adhesive capability, easily clustering (not good for WST-1 assays measurement), and difficultly being cultured (Figure S3), so we decided to only use SF8628 for subsequent experiments. We next sought to determine the mechanisms for anti-DIPG activity of ABC294640. Using flow cytometry, we found that ABC294640 treatment induced apoptosis of DIPG cells in a dose-dependent fashion, including the increased subpopulation of both early (Annexin $\mathrm{V}+/ \mathrm{PI}-$ ) and late (Annexin V+/PI+) apoptotic cells (Figure 1B-C). We also observed the increased cleavage of caspases-3 and PARP, and the expression of pro-apoptotic protein, Bax, from ABC294640treated DIPG cells (Figure 1D). In contrast, we observed a decreased expression of X-linked inhibitor of apoptosis protein (XIAP), which is a physiologic substrate of Akt, and its function is stabilized to inhibit programmed cell death and having a direct effect on caspases [36]. These results are consistent with the notion that ABC294640 exerts an antiproliferative effect on DIPG cells by inducing programmed cell death.

\section{ABC294640 induces intracellular ceramides production from DIPG cells by lipidomics analysis}

Next, we sought to determine if these effects were related to changes in sphingolipid metabolism. Mass spectrometric (MS)-based lipidomics analysis were used to quantify and compare intracellular levels of bioactive ceramide species in the SF8628 cell line treated with or without $\mathrm{ABC} 294640$. We found that ABC294640 increased total levels of intracellular ceramides ( $\sim 8$ folds) in DIPG cells when compared to 
the vehicle group. The lipidomics analysis showed that most of individual ceramide species including those from C14-Cer to C26-Cer were upregulated in ABC294640-treated DIPG cells, although the extent of the increase varies among the ceramide species (Figure 1E-F). The composition and proportion of ceramide species within SF8628 cells with or without ABC294640 treatment were calculated (Figure S4). The predominant ceramide signature within DIPG cells included C16-, C22- and C24:1-Cer species. Interestingly, we found that $\mathrm{ABC} 294640$ treatment did not significantly alter the proportion of ceramide species in DIPG cells, which is different from what we have observed in virus-associated tumors and lung cancers $[26,28,29]$.

Table 1. The top 20 candidate genes upregulated or downregulated in DIPG cells treated by ABC294640

\begin{tabular}{|c|c|c|}
\hline Gene Symbol & Description & Ratio \\
\hline TM4SF19-TCTEX1D2 & TM4SF19-TCTEX1D2 & 203.2956944 \\
\hline SERPINB2 & Serpin family B member 2 & 198.8279343 \\
\hline CXCL8 & $\mathrm{C}-\mathrm{X}-\mathrm{C}$ motif chemokine ligand 8 & 175.8729164 \\
\hline AP003419.11 & AP003419.11/novel protein & 166.9185386 \\
\hline KRT34 & Keratin 34 & 163.7514827 \\
\hline RP11-466H18.1 & Ribosomal protein L36a pseudogene & 153.5685184 \\
\hline ANKRD22 & Ankyrin repeat domain 22 & 139.5586948 \\
\hline CH507-9B2.4 & CH507-9B2.4/novel protein & 92.99857464 \\
\hline SPINK1 & Serine peptidase inhibitor, Kazal type 1 & 88.91478078 \\
\hline SLC7A11-AS1 & SLC7A11 antisense RNA 1 & 83.299466 \\
\hline ANGPTL4 & Angiopoietin like 4 & 79.47843571 \\
\hline CORO7-PAM16 & CORO7-PAM16 & 75.18388531 \\
\hline CTD-2066L21.3 & CTD-2066L21.3/lincRNA & 71.37901957 \\
\hline AC068533.7 & AC068533.7/novel protein & 70.82918348 \\
\hline RP11-1151B14.4 & Novel transcript, antisense to ALPK2 & 62.40569457 \\
\hline CXorf67 & Chromosome $X$ open reading frame 67 & 58.1829534 \\
\hline IL36B & Interleukin 36 , beta & 53.78426467 \\
\hline TNFSF12-TNFSF13 & TNFSF12-TNFSF13 & 51.2770121 \\
\hline GPR78 & G protein-coupled receptor 78 & 51.25501866 \\
\hline RP11-613M10.8 & RP11-613M10.8/novel protein & 50.4192678 \\
\hline COL3A1 & Collagen type III alpha 1 chain & 0.003891426 \\
\hline IFITM1 & $\begin{array}{l}\text { Interferon induced transmembrane } \\
\text { protein } 1\end{array}$ & 0.006783706 \\
\hline LGALS2 & Galectin 2 & 0.009437712 \\
\hline HEYL & $\begin{array}{l}\text { Hes related family bHLH transcription } \\
\text { factor with YRPW motif-like }\end{array}$ & 0.0097645 \\
\hline RP11-588K22.2 & Novel transcript, overlapping GUCY1A3 & 0.01022648 \\
\hline AC093850.2 & AC093850.2/novel transcript & 0.011349859 \\
\hline DUSP27 & Dual specificity phosphatase 27 & 0.012750501 \\
\hline TLL1 & Tolloid like 1 & 0.012939696 \\
\hline RP11-680F20.6 & $\begin{array}{l}\text { V-set and immunoglobulin } \\
\text { domain-containing protein 10-like }\end{array}$ & 0.013194198 \\
\hline C1QTNF3-AMACR & C1QTNF3-AMACR & 0.013937263 \\
\hline APOC1 & Apolipoprotein $\mathrm{C} 1$ & 0.014431779 \\
\hline $\mathrm{CDH} 23$ & Cadherin related 23 & 0.015076107 \\
\hline TEX41 & Testis expressed 41 & 0.015646885 \\
\hline KAL1 & Anosmin 1 & 0.015662941 \\
\hline COL5A3 & Collagen type $\mathrm{V}$ alpha 3 chain & 0.016356182 \\
\hline DLX3 & Distal-less homeobox 3 & 0.017533291 \\
\hline RP11-598P20.5 & RP11-598P20.5/novel protein & 0.017621652 \\
\hline ZMYND15 & Zinc finger MYND-type Containing 15 & 0.017651757 \\
\hline MUC7 & Mucin 7 , secreted & 0.017651757 \\
\hline
\end{tabular}

Transcriptomic analysis of gene profiling altered within ABC294640-treated DIPG cells

To determine the global metabolic and cellular changes induced by ABC294640, we compared the global gene profile altered between vehicle- and ABC294640-treated tumor cells by using RNASequencing (Illumina) analysis. We first found 485 genes significantly upregulated and 782 genes significantly downregulated ( $\geq 2$ folds and FDR $<0.05$ ) in ABC294640-treated SF8628 cell line, respectively (Figure 2A). The top 20 significantly upregulated and downregulated candidates were listed in Table 1. Interestingly, most of these candidates, their functions have never been reported in DIPG cells. Ingenuity pathway analysis (IPA) analysis of altered gene profiling indicated that many canonical pathways and disease_function categories within DIPG cells were affected by ABC294640 treatment (Figure 2B-C). For example, several canonical pathways related to cancer cell survival, such as retinoic acid mediated apoptosis signaling, cyclins and cell cycle regulation, and calcium signaling were impacted by treatment with ABC294640. Since ABC294640 is a selective SphK inhibitor, it is not surprising to see several fatty acid/ lipid synthesis categories negatively regulated by ABC294640.

\section{Sphingolipid metabolism regulation of IFITMI and KALI which are required for DIPG cell survival}

To functionally validate those significantly altered genes within ABC294640-treated DIPG cells discovered by RNA-Sequencing, we selected two candidates, IFITM1 and KAL1, from the list. Interferon-induced transmembrane protein 1 (IFITM1) is originally identified as part of membrane complexes transducing homotypic adhesion signals in lymphocytes [37,38]. The expression of IFITM1 is induced by IFN- $\alpha$ and/or IFN- $\gamma$ (to a lesser extent), and has been reported in several types of cancer cells $[39,40]$. Anosmin-1, encoded by the KAL1 gene, is an important extracellular matrix (ECM) component that plays essential roles in the establishment of olfactory and gonadotrophin-releasing hormone (GNRH) neurons during early brain development. Loss-offunction mutations of KAL1 results in Kallmann syndrome with delayed puberty and anosmia [41]. However, their functions in DIPG cells remain unclear.

We observed the downregulation of both IFITM1 and KAL1 in DIPG cells treated with ABC294640 (Table 1). We next found that silencing of either IFITM1 or KAL1 by RNAi effectively repressed DIPG cell growth, inducing tumor cell apoptosis in a manner similar to treatment with ABC294640 (Figure 
3A-C). RNAi against either IFITM1 or KAL1 also inhibited anchorage-independent growth of DIPG cells (Figure 3D-E), again consistent with the results obtained in experiments with the SphK inhibitor.

Currently, a DIPG clinical database is not publically available. Recently, we have obtained the permission to access RNA-Sequencing data of DIPG tumor samples and paired normal brain tissues from the NIH Genotypes and Phenotypes (dbGaP) database. Here we found that both IFITM1 and KAL1 expression were higher in DIPG tumor samples than in their paired normal brain tissues, although only KAL1 proved to be statistically significant probably due to the small number of collection (Figure 3F). Together, these data support a role for IFITM1 and KAL1 in promoting DIPG survival or growth.

To further understand the connection of IFITM1 and KAL1 with sphingolipid metabolism in DIPG cells, we silenced SphK1 and SphK2 in SF8628, two of major enzymes in sphingolipid metabolism using RNAi, respectively. We found that knockdown of either SphK1 or SphK2 effectively reduced IFITM1 and KAL1 expression (Figure 3G) indicating sphingolipid metabolism indeed regulates these cellular genes expression and functions to determine tumor cell survival.

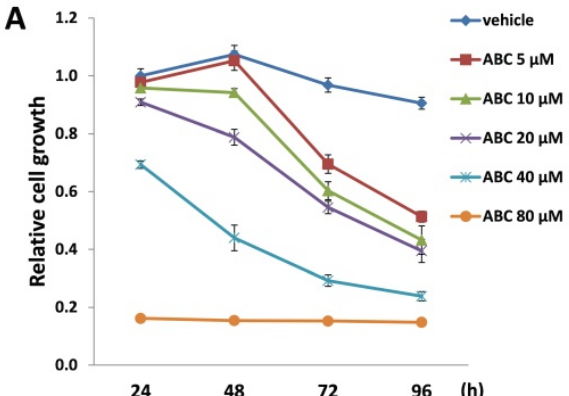

D

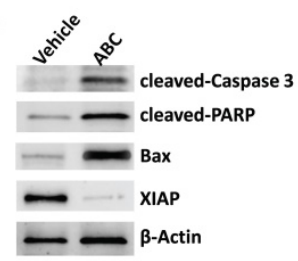

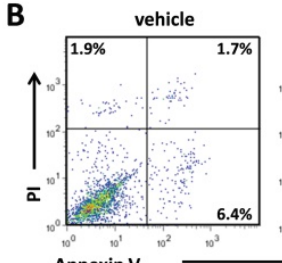
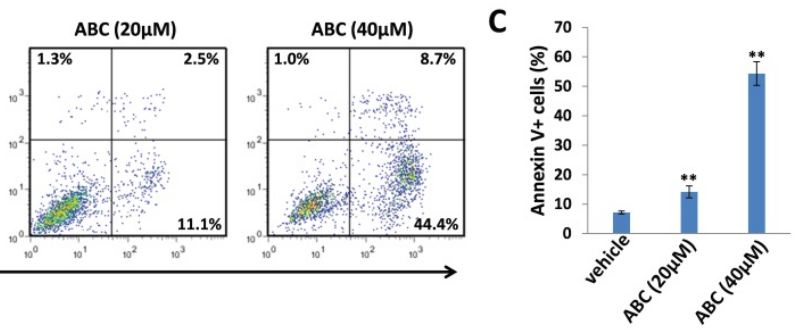

$\mathbf{F}$

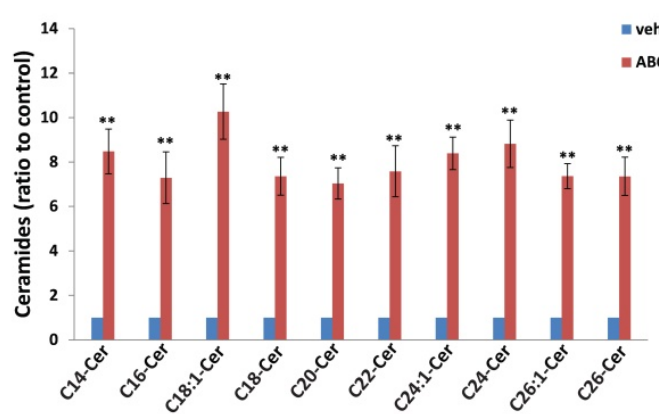

Figure 1. ABC294640 treatment effectively reduces DIPG proliferation through inducing tumor cell apoptosis and intracellular ceramides production. (A) DIPG cell line SF8628 were treated with the indicated concentrations of ABC294640 or vehicle for $24-96 \mathrm{~h}$. The cell proliferation status was then examined using the WST-1 cell proliferation assays (Roche). (B-C) SF8628 were treated with the indicated concentrations of ABC294640 or vehicle for $24 \mathrm{~h}$. Cell viability and apoptosis were then measured by Annexin V-PI staining and flow cytometry analysis. Error bars represent S.D. for 3 independent experiments. (D) Protein expression was detected using immunoblots. (E-F) SF8628 were treated with $40 \mu \mathrm{M}$ of $\mathrm{ABC} 294640$ or vehicle for $24 \mathrm{~h}$. Ceramide species and total levels were then quantified using lipidomics analyses as described in the Methods. Error bars represent S.D. for 2 independent experiments. $* *=p<0.01$.

A

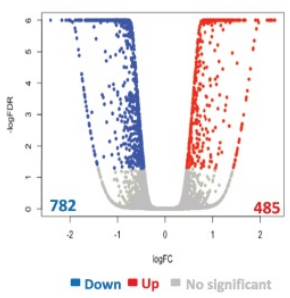

C

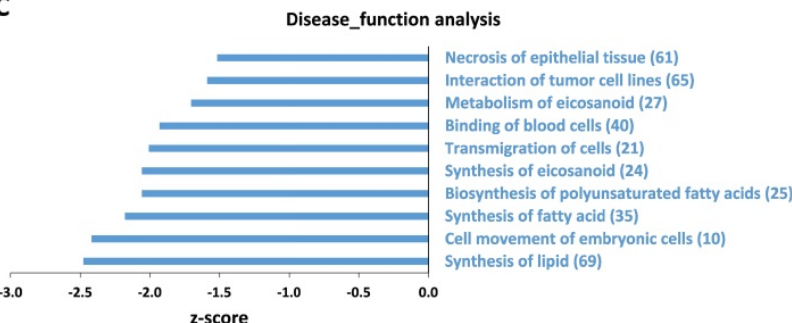

B

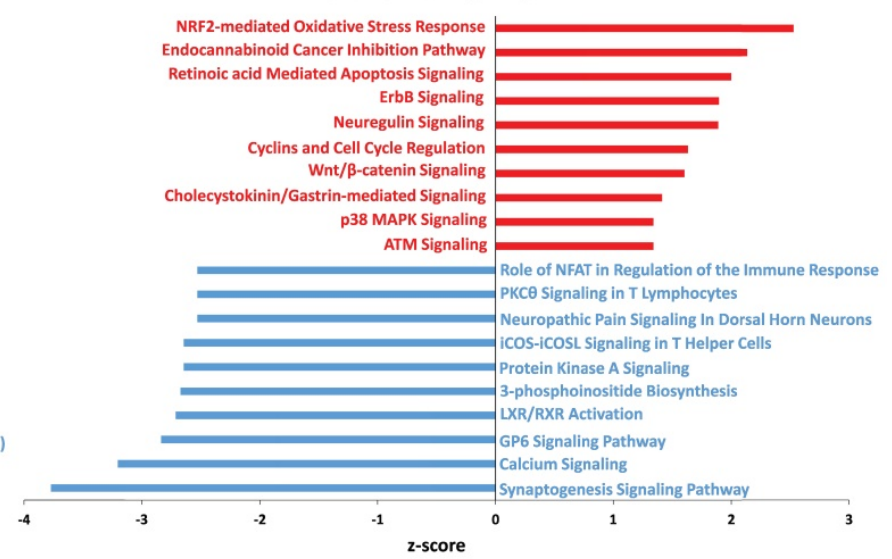

Figure 2. Ingenuity pathway analysis (IPA) of global gene profile altered by ABC294640 from DIPG cells. (A) The RNA-Sequencing (Illumina) was used to investigate the transcriptome change between ABC294640 and vehicle treated DIPG SF8628 cells. The significantly changed genes (expression change $\geq 2$-fold and $P<0.05$ ) were shown in the Volcano plot panels. (B-C) The top 10 activated (red) and 10 inhibited (blue) canonical pathways (or Disease functions) discovered in the ABC294640-treated cells by the IPA. The pathways (B)/functions (C) were ranked by the Z-score. The Z-score is a value calculated by the Z-score algorithm of the IPA. The Z-score is utilized to predict the direction of change for a biological function: if it is increased, the Z-score is $>0$; if it is decreased, the Z-score is $<0$. 
A

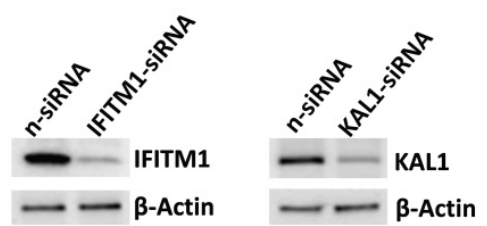

D

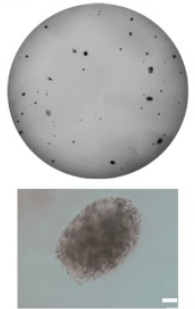

n-siRNA
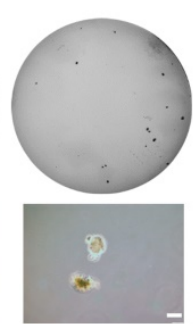

IFITM1-siRNA

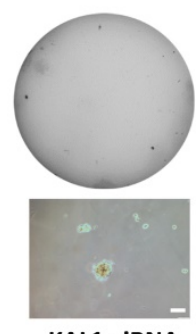

KAL1-siRNA

E

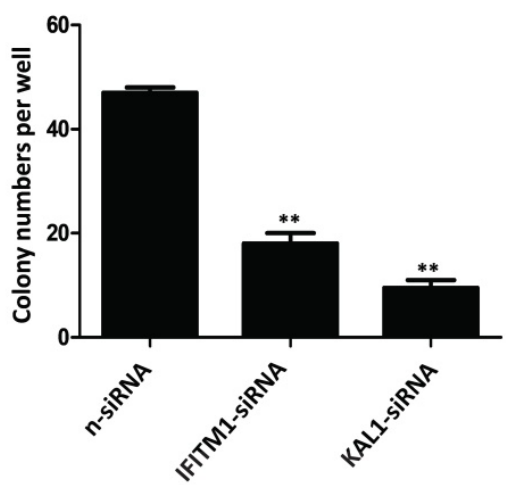

B

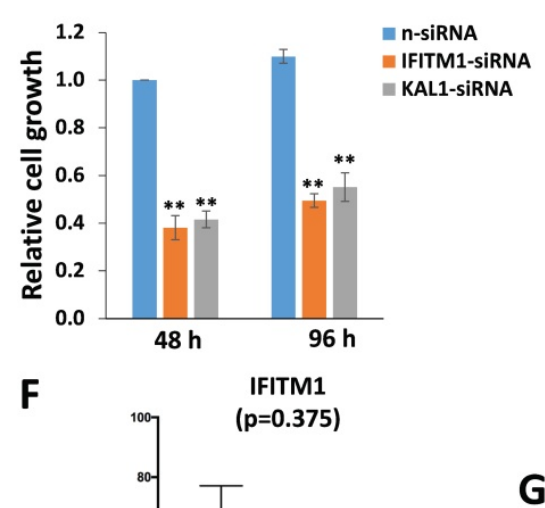

G
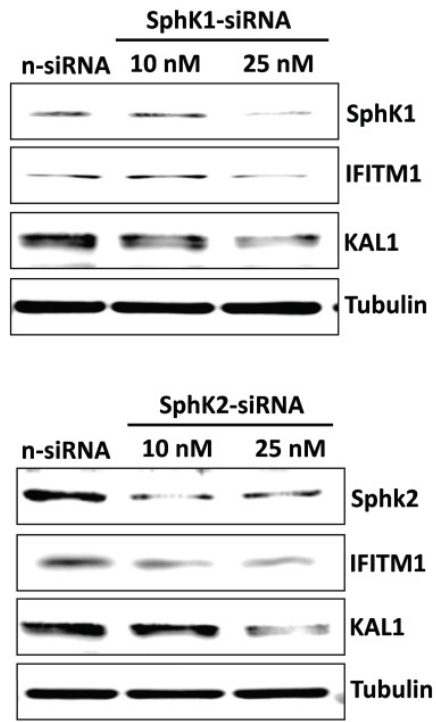

Figure 3. Sphingolipid metabolism regulation of IFITMI and KALI expression is essential for DIPG cell survival and growth. (A-C) SF8628 were transfected with IFITM I-siRNA, KALI-siRNA or non-target control siRNA (n-siRNA) for 48-96 h, then the protein expression, cell proliferation and apoptosis were measured as described above. (D-E) The anchorage-independent growth ability was determined using the soft agar assays. Error bars represent S.D. for 3 independent experiments. $* *=p<0.01$. (F) The expression of IFITMI and KALI in 10 DIPG and paired normal brain tissues. RPKM (Reads Per Kilobase of transcript per Million mapped reads) values were calculated using reads across the gene exons. Data were represented as the mean (+/-) SEM. (G) SF8628 were transfected with SphKI-siRNA, SphK2-siRNA or non-target control siRNA (n-siRNA) for $72 \mathrm{~h}$, then the protein expression was measured by immunoblots.

\section{Discussion}

As a rare but highly aggressive pediatric cancer, DIPG is currently lacks an effective treatment, making prognosis very poor. Our efforts seek to identify new therapeutic targets and develop promising strategies for maximally prolonging the survival of patients. In the current study, we used one of few commercially available DIPG patient-derived cell lines (SF8628) to determine if targeting sphingolipid metabolism is a useful strategy for improving patient outcomes. We found that treatment with ABC294640, the only firstin-class orally available inhibitor of SphK, selectively repressed DIPG cell growth through inducing intracellular pro-apoptotic ceramides production and tumor cell apoptosis. In general, there are three major ceramide generation pathways: the sphingomyelinase pathway (sphingomyelin $\rightarrow$ ceramide); the de novo pathway (3-keto-dihydrosphingosine $\rightarrow$ dihydro sphingosine $\rightarrow$ dihydroceramide $\rightarrow$ ceramide); and the
Salvage pathway (S1P $\rightarrow$ sphingosine $\rightarrow$ ceramide) $[16,42]$. MS-based lipidomics analyses will be used to further determine which pathways mediate intracellular ceramides production from ABC294640treated DIPG cells. Ceramide synthases (CerSs) are the enzymes responsible for ceramide generation in the de novo and Salvage pathways. Currently, six different CerSs have been identified in mammary cells, CerS1-6 [43], and different isoforms of CerS generate an array of ceramide species with distinct length of fatty acid chain [44]. Our previous study showed that ABC294640 treatment increased the gene transcription and protein expression for CerS2, CerS4 and CerS6 from some virus-associated lymphomas [26]. However, the signature of ceramide species and regulation of ceramide production are potentially differentiated in different types of cancer cells. For example, we found that ABC294640 treatment did not alter the proportion of ceramide species in DIPG cells, in contrast to the significant changes we observed 
previously in virus-associated tumors and lung cancers $[26,28,29]$. In addition, we previously reported that ABC294640 treatment induced dihydro(dh)ceramide production in virus-associated tumors and lung cancers, which also contributed to "killing" of tumor cells $[20,28,29]$. In contrast, we found low or undetectable levels of dh-ceramide species in DIPG cells even after exposure to ABC294640 (data not shown), implying that dh-ceramides are not involved in ABC294640-mediated DIPG cell death.

Although the functional role of sphingolipid metabolism (especially ceramide production) and targeted therapy have not been reported in DIPG, there are some related reports in other brain tumors. One recent study reported that the overexpression of CerS1 increased C18-Cer level and led to lethal autophagy in human glioma cells [45]. Another recent study revealed that co-delivery of tumor-derived exosomes with a-galactosylceramide (a-GalCer) on a dendritic cell (DC)-based vaccine showed excited effects on glioblastoma immunotherapy, inducing strong activation of tumor-specific cytotoxic $\mathrm{T}$ lymphocytes, synergistically breaking immune tolerance and improving the immunosuppressive environment in vivo [46]. In one review article, Sordillo et al postulated that some chemotherapeutic agents or radiotherapy may induce short-term responses in glioblastoma patients by increasing ceramide levels, however, the SphK may cause the increased ceramide to be metabolized to S1P, therefore restoring the abnormally high S1P to ceramide balance and representing part of the reason for the nearly $100 \%$ recurrence rate of glioblastoma [47]. Thus, the use of maintenance therapy with a SphK inhibitor in patients with glioblastoma who have tumor reduction or stable disease after therapy should be investigated and considered.

In addition to the regulation of intracellular ceramides production, sphingolipid metabolism actually regulates many other cellular factors related to cell survival or death. Our recent transcriptomic analyses revealed that a subset of tumor suppressor genes ( $\sim 25$ genes) were significantly upregulated in virus-associated lymphomas by exogenous dhC16-Cer [27], which may control tumor cell growth. In this study, we have identified new genes within DIPG affected by ABC294640. Two of these genes, IFITM1 and KAL1, were selected and found to have a strong impact on DIPG cell survival with clinical relevance. Interestingly, Balbous et al reported IFITM1 as one of mesenchymal glioma stem cell makers responsible for cell invasion and gliomasphere initiation, which also revealed strong correlation with overall survival of glioblastoma patients [48]. Several studies reported overexpression of IFITM1 in some types of tumors, such as colorectal, gastrointestinal and breast cancers $[49,50]$. Furthermore, these studies demonstrated a positive correlation between IFITM1 overexpression and tumor progression, too. For another gene, KAL1, Choy et al found that its mRNA level was significantly upregulated in high-grade primary brain tumors when compared to the normal brain and/or low-grade tumors [51]. They also found that KAL1 enhanced glioblastoma cells proliferation and motility in vitro, through forming a complex with integrin $\beta 1$ induced downstream signaling, and modulating cell adhesion [51]. Interestingly, we found that both SphK1 and SphK2 could regulate IFITM1 and KAL1 expression, although ABC294640 has much less impact on SphK1 when compared to SphK2. Thus our data demonstrate that sphingolipid metabolism indeed regulates the expression of other cellular factors related to DIPG survival such as IFITM1 and KAL1, while the regulatory mechanisms still require further investigation.

In conclusion, our data for the first time demonstrate sphingolipid metabolism controls DIPG cell survival, which may represent a promising target against this rare but deadly pediatric brain tumor. Our findings also provide new evidence that sphingolipid metabolism regulates additional cellular factors to affect DIPG cell survival and growth. Although its promise in DIPG treatment, there are still many challenging for developing sphingolipid metabolism targeted therapy including ABC294640. One of remained questions is the permeability and uptake of ABC294640 to blood-brain barrier (BBB) and blood-tumor barrier (BTB), which has never been tested before. Also, the in vivo efficacy of ABC294640 or other sphingolipid-related agents need to be assessed by using DIPG intracranial animal models in future studies.

\section{Supplementary Material}

Supplementary figures and tables.

http://www.jcancer.org/v11p4683s1.pdf

\section{Acknowledgements}

We thank Dr. Jovanny Zabaleta at LSUHSC to help the RNA-Sequencing and Dr. Robert Eoff at UAMS to help discussion and edition during the manuscript preparation. This work is supported by the Arkansas Bioscience Institute, the major research component of the Arkansas Tobacco Settlement Proceeds Act of 2000 (to Z.Q.). S.M. is supported by NIH 1R01 HL146713. Z.L. is supported by a NIH COBRE grant (P20 GM121288), a Tulane school of medicine faculty research pilot grant, and a Carol Lavin Bernick faculty grant. The funders had no role 
in study design, data collection and analysis, decision to publish, or preparation of the manuscript.

\section{Competing Interests}

The authors have declared that no competing interest exists.

\section{References}

1. Long W, Yi Y, Chen S, Cao Q, Zhao W, Liu Q. Potential New Therapies for Pediatric Diffuse Intrinsic Pontine Glioma. Front Pharmacol. 2017; 8: 495.

2. Mohammad F, Weissmann S, Leblanc B, Pandey DP, Hojfeldt JW, Comet I, et al. EZH2 is a potential therapeutic target for H3K27M-mutant pediatric gliomas. Nat Med. 2017; 23: 483-+.

3. Lapin DH, Tsoli M, Ziegler DS. Genomic Insights into Diffuse Intrinsic Pontine Glioma. Frontiers in Oncology. 2017; 7.

4. Piunti A, Hashizume R, Morgan MA, Bartom ET, Horbinski CM, Marshall SA, et al. Therapeutic targeting of polycomb and BET bromodomain proteins in diffuse intrinsic pontine gliomas. Nat Med. 2017; 23: 493-+.

5. Gwak HS, Park HJ. Developing chemotherapy for diffuse pontine intrinsic gliomas (DIPG). Crit Rev Oncol Hemat. 2017; 120: 111-9.

6. Pollack IF, Agnihotri S, Broniscer A. Childhood brain tumors: current management, biological insights, and future directions. J Neurosurg-Pediatr. 2019; 23: 261-73.

7. Chen J, Lin Z, Barrett L, Dai L, Qin Z. Identification of new therapeutic targets and natural compounds against diffuse intrinsic pontine glioma (DIPG). Bioorganic Chemistry. 2020; 99: 103847.

8. Bellat V, Alcaina Y, Tung C-H, Ting R, Michel A, Souweidane M, et al. A Combined Approach of Convection-Enhanced Delivery of Peptide Nanofiber Reservoir to Prolong Delivery of DM1 for Diffuse Intrinsic Pontine Glioma Treatment. Neuro-Oncology. 2020.

9. Boulet MHC, Marsh LK, Howarth A, Woolman A, Farrer NJ. Oxaliplatin and $[\mathrm{Pt}(\mathrm{R}, \mathrm{R}-\mathrm{DACH})($ panobinostat-2H)] show nanomolar cytotoxicity towards diffuse intrinsic pontine glioma (DIPG). Dalton Transactions. 2020; 49: 5703-10

10. Meel MH, de Gooijer MC, Metselaar DS, Sewing ACP, Zwaan K, Waranecki $\mathrm{P}$, et al. Combined therapy of AXL and HDAC inhibition reverses mesenchymal transition in diffuse intrinsic pontine glioma. Clin Cancer Res. 2020: clincanres.3538.2019.

11. Kluiver TA, Alieva M, van Vuurden DG, Wehrens EJ, Rios AC. Invaders Exposed: Understanding and Targeting Tumor Cell Invasion in Diffuse Intrinsic Pontine Glioma. Frontiers in Oncology. 2020; 10.

12. Bailey CP, Figueroa M, Mohiuddin S, Zaky W, Chandra J. Cutting Edge Therapeutic Insights Derived from Molecular Biology of Pediatric High-Grade Glioma and Diffuse Intrinsic Pontine Glioma (DIPG). Bioengineering. 2018; 5: 88

13. Su JM, Murray JC, McNall-Knapp RY, Bowers DC, Shah S, Adesina AM, et al. A phase 2 study of valproic acid and radiation, followed by maintenance valproic acid and bevacizumab in children with newly diagnosed diffuse intrinsic pontine glioma or high-grade glioma. Pediatr Blood Cancer. 2020; 67: e28283.

14. Ogretmen B, Hannun YA. Biologically active sphingolipids in cancer pathogenesis and treatment. Nat Rev Cancer. 2004; 4: 604-16.

15. Cuvillier O, Pirianov G, Kleuser B, Vanek PG, Coso OA, Gutkind JS, et al. Suppression of ceramide-mediated programmed cell death by sphingosine-1-phosphate. Nature. 1996; 381: 800-3.

16. Liu JW, Beckman BS, Foroozesh M. A review of ceramide analogs as potential anticancer agents. Future Med Chem. 2013; 5: 1405-21.

17. Saddoughi SA, Ogretmen B. Diverse Functions of Ceramide in Cancer Cell Death and Proliferation. Adv Cancer Res. 2013; 117: 37-58.

18. French KJ, Schrecengost RS, Lee BD, Zhuang Y, Smith SN, Eberly JL, et al. Discovery and evaluation of inhibitors of human sphingosine kinase. Cancer Res. 2003; 63: 5962-9.

19. French KJ, Zhuang Y, Maines LW, Gao P, Wang WX, Beljanski V, et al. Pharmacology and Antitumor Activity of ABC294640, a Selective Inhibitor of Sphingosine Kinase-2. J Pharmacol Exp Ther. 2010; 333: 129-39.

20. Qin ZQ, Dai L, Trillo-Tinoco J, Senkal C, Wang WX, Reske T, et al Targeting Sphingosine Kinase Induces Apoptosis and Tumor Regression for KSHV-Associated Primary Effusion Lymphoma. Mol Cancer Ther. 2014; 13: 154-64.

21. Venant H, Rahmaniyan M, Jones EE, Lu P, Lilly MB, Garrett-Mayer E, et al. The Sphingosine Kinase 2 Inhibitor ABC294640 Reduces the Growth of Prostate Cancer Cells and Results in Accumulation of
Dihydroceramides In Vitro and In Vivo. Mol Cancer Ther. 2015; 14: 2744-52.

22. Xun C, Chen MB, Qi L, Zhang TN, Peng X, Ning L, et al. Targeting sphingosine kinase 2 (SphK2) by ABC294640 inhibits colorectal cancer cell growth in vitro and in vivo. J Exp Clin Canc Res. 2015; 34.

23. Lewis CS, Voelkel-Johnson C, Smith CD. Suppression of c-Myc and RRM2 expression in pancreatic cancer cells by the sphingosine kinase-2 inhibitor ABC294640. Oncotarget. 2016; 7: 60181-92.

24. Ding XW, Chaiteerakij R, Moser CD, Shaleh H, Boakye J, Chen G, et al. Antitumor effect of the novel sphingosine kinase 2 inhibitor ABC294640 is enhanced by inhibition of autophagy and by sorafenib in human cholangiocarcinoma cells. Oncotarget. 2016; 7: 20080-92.

25. Britten CD, Garrett-Mayer E, Chin SH, Shirai K, Ogretmen B, Bentz TA, et al. A Phase I Study of ABC294640, a First-in-Class Sphingosine Kinase-2 Inhibitor, in Patients with Advanced Solid Tumors. Clin Cancer Res. 2017; 23: 4642-50.

26. Dai L, Trillo-Tinoco J, Bai AP, Chen YH, Bielawski J, Del Valle L, et al. Ceramides promote apoptosis for virus-infected lymphoma cells through induction of ceramide synthases and viral lytic gene expression. Oncotarget. 2015; 6: 24246-60.

27. Cao YY, Qiao J, Lin Z, Zabaleta J, Dai L, Qin ZQ. Up-regulation of tumor suppressor genes by exogenous dhC16-Cer contributes to its anti-cancer activity in primary effusion lymphoma. Oncotarget. 2017; 8: 15220-9.

28. Dai L, Bai AP, Smith CD, Rodriguez PC, Yu FY, Qin ZQ. ABC294640, A Novel Sphingosine Kinase 2 Inhibitor, Induces Oncogenic Virus-Infected Cell Autophagic Death and Represses Tumor Growth. Mol Cancer Ther. 2017; 16: 2724-34

29. Dai L, Smith CD, Foroozesh M, Miele L, Qin ZQ. The sphingosine kinase 2 inhibitor ABC294640 displays anti-non-small cell lung cancer activities in vitro and in vivo. Int J Cancer. 2018; 142: 2153-62.

30. Dai L, Trillo-Tinoco J, Chen YH, Bonstaff K, Del Valle L, Parsons C, et al. CD147 and downstream ADAMTSs promote the tumorigenicity of Kaposi's sarcoma-associated herpesvirus infected endothelial cells. Oncotarget. 2016; 7: 3806-18.

31. Bielawski J, Szulc ZM, Hannun YA, Bielawska A. Simultaneous quantitative analysis of bioactive sphingolipids by high-performance liquid chromatography-tandem mass spectrometry. Methods. 2006; 39: 82-91.

32. Kheir F, Zhao MM, Strong MJ, Yu Y, Nanbo A, Flemington EK, et al. Detection of Epstein-Barr Virus Infection in Non-Small Cell Lung Cancer. Cancers. 2019; 11.

33. Mueller S, Hashizume R, Yang XD, Kolkowitz I, Olow AK, Phillips J, et al. Targeting Wee1 for the treatment of pediatric high-grade gliomas. Neuro-Oncology. 2014; 16: 352-60.

34. Lewis PW, Allis CD. Poisoning the "histone code" in pediatric gliomagenesis. Cell Cycle. 2013; 12: 3241-2.

35. Wan YCE, Liu J, Chan KM. Histone H3 Mutations in Cancer. Curr Pharmacol Rep. 2018; 4: 292-300.

36. Dan HC, Sun M, Kaneko S, Feldman RI, Nicosia SV, Wang HG, et al. Akt phosphorylation and stabilization of X-linked inhibitor of apoptosis protein (XIAP). J Biol Chem. 2004; 279: 5405-12.

37. Lewin AR, Reid LE, Mcmahon M, Stark GR, Kerr IM. Molecular Analysis of a Human Interferon-Inducible Gene Family. Eur J Biochem. 1991; 199: 417-23.

38. Deblandre GA, Marinx OP, Evans SS, Majjaj S, Leo O, Caput D, et al. Expression Cloning of an Interferon-Inducible 17-Kda Membrane-Protein Implicated in the Control of Cell-Growth. J Biol Chem. 1995; 270: 23860-6.

39. Yang Y, Lee JH, Kim KY, Song HK, Kim JK, Yoon SR, et al. The interferon-inducible 9-27 gene modulates the susceptibility to natural killer cells and the invasiveness of gastric cancer cells. Cancer Lett. 2005; 221: 191-200.

40. Brass AL, Huang IC, Benita Y, John SP, Krishnan MN, Feeley EM, et al. The IFITM Proteins Mediate Cellular Resistance to Influenza A H1N1 Virus, West Nile Virus, and Dengue Virus. Cell. 2009; 139: 1243-54.

41. Schwanzel-Fukuda M, Bick D, Pfaff DW. Luteinizing hormone-releasing hormone (LHRH)-expressing cells do not migrate normally in an inherited hypogonadal (Kallmann) syndrome. Molecular Brain Research. 1989; 6: 311-26

42. Hannun YA, Obeid LM. Principles of bioactive lipid signalling: lessons from sphingolipids. Nat Rev Mol Cell Bio. 2008; 9: 139-50.

43. Stiban J, Tidhar R, Futerman AH. Ceramide Synthases: Roles in Cell Physiology and Signaling. Adv Exp Med Biol. 2010; 688: 60-71.

44. Hannun YA, Obeid LM. Many Ceramides. J Biol Chem. 2011; 286: 27855-62.

45. Wang Z, Wen LJ, Zhu F, Wang YP, Xie Q, Chen ZJ, et al. Overexpression of ceramide synthase 1 increases C18-ceramide and leads to lethal autophagy in human glioma. Oncotarget. 2017; 8: 104022-36. 
46. Liu HY, Chen L, Liu JL, Meng HX, Zhang R, Ma L, et al. Co-delivery of tumor-derived exosomes with alpha-galactosylceramide on dendritic cell-based immunotherapy for glioblastoma. Cancer Lett. 2017; 411: 182-90.

47. Sordillo LA, Sordillo PP, Helson L. Sphingosine Kinase Inhibitors as Maintenance Therapy of Glioblastoma After Ceramide-Induced Response. Anticancer Res. 2016; 36: 2085-95.

48. Balbous A, Cortes U, Guilloteau K, Villalva C, Flamant S, Gaillard A, et al. A mesenchymal glioma stem cell profile is related to clinical outcome. Oncogenesis. 2014; 3 .

49. Lee J, Goh SH, Song N, Hwang JA, Nam S, Choi IJ, et al. Overexpression of IFITM1 Has Clinicopathologic Effects on Gastric Cancer and Is Regulated by an Epigenetic Mechanism. Am J Pathol. 2012; 181: 43-52.

50. Andreu P, Colnot S, Godard C, Laurent-Puig P, Lamarque D, Kahn A, et al. Identification of the IFITM family as a new molecular marker in human colorectal tumors. Cancer Res. 2006; 66: 1949-55.

51. Choy CT, Kim H, Lee JY, Williams DM, Palethorpe D, Fellows G, et al. Anosmin-1 contributes to brain tumor malignancy through integrin signal pathways. Endocr-Relat Cancer. 2014; 21: 85-99. 